\title{
INCIDENCE OF NON-B SUBTYPES OF HIV-1 IN GALICIA, SPAIN: HIGH FREQUENCY AND DIVERSITY OF HIV-1 AMONG MEN WHO HAVE SEX WITH MEN
}

\author{
M T Cuevas ${ }^{1}$, A Fernández-García ${ }^{1}$, A Sánchez-Garcia ${ }^{1}$, M González-Galeano ${ }^{1}$, M Pinilla ${ }^{1}$, M Sánchez- Martínez ${ }^{1}$, V García ${ }^{1}$, L \\ Pérez-Álvarez (lperezal@isciii.es)1, Study group of HIV-1 newly diagnosed patients in Galicia, Spain² \\ 1. HIV Biology and Variability Department, CNM, Instituto de Salud Carlos III, Majadahonda, Madrid, Spain \\ 2. Members of the Study group are listed at the end of the article \\ 3. Sector of Laboratory Assistance, National Institute of Health, Lisbon, Portugal
}

This article was published on 26 November 2009.

Citation style for this article: Cuevas MT, Fernández-García A, Sánchez-García A, González-Galeano M, Pinilla M, Sánchez-Martínez M, García V, Pérez-Álvarez L, Study group of HIV-1 newly diagnosed patients in Galicia, Spain. Incidence of non-B subtypes of HIV-1 in Galicia, Spain: high frequency and diversity of HIV-1 among men who have sex with men. Euro Surveill. 2009;14(47):pii=19413. Available online: http://www.eurosurveillance.org/ViewArticle.aspx?ArticleId=19413

An increase in HIV transmission among men who have sex with men (MSM) has been reported in eight regions of Spain from 2003 to 2007. In order to study the incidence of HIV-1 genetic forms in Galicia, northwest of Spain, in particular the spread of HIV-1 variants among MSM, 93 newly diagnosed HIV-1 patients, including those with acute and recently acquired infections, were studied for a year from August 2008 to August 2009. Thirty eight (41\%) were MSM. Of them, nine (24\%) were infected by non-B viruses, including seven different genetic forms. The analysis of transmission clusters showed that $23(60 \%)$ MSM grouped in different clusters and mostly in large clusters. Resistance mutations were detected in six (16\%) MSM.

\section{Introduction}

Subtype B of HIV-1 is the most prevalent in Western Europe, including Spain, however, an increase in the circulation of non-B subtypes has been observed in recent years [1,2]. Another trend is the increase in HIV transmission among MSM, a phenomenon recently described in eight countries in North America, Western Europe and Australia [3].

The objective of this survey was to study the incidence of HIV-1 genetic forms in Galicia, north-western Spain, in particular the spread of HIV-1 variants among MSM.

\section{Patients and methods}

A total of 93 newly diagnosed HIV-1 patients, 72 males and 21 females who attended six hospitals of the Public Health Service of Galicia, Spain, were recruited from August 2008 to August 2009. Seventy nine (85\%) were born in Spain and 14 (15\%) were foreigners. Sixteen patients (17\%) were at the early phase of infection, including nine with acute and seven with recently acquired infection. Transmission route was mainly by sexual contact (84\%), less frequent was injecting drug use (16\%).

Of the patients included in the study 38 (41\%) were MSM. Of them, 32 were born in Spain. Their average age was 30.6 years (excluding a 73-year-old man), lower than the average of age of heterosexual patients (44.3 years).
RNA was extracted from plasma and amplified by RT-PCR in pol region, corresponding to protease and partial reverse transcriptase (PR-RT), (HXB2 positions 2107 to 3630). Sequences were assembled using Seqman software and edited with Bioedit program. For subtype assignment, phylogenetic trees were done with Mega software (http://www.megasoftware.net/) using neighbour joining method and Rega software was also used (http://dbpartners. stanford.edu/RegaSubtyping/). Resistance mutations were defined following Standford Database criteria (http://hivdb.stanford.edu/). Transmission clusters were also analysed and defined as those sequences which grouped together with a bootstrap value $>=70 \%$, following the criteria of Hillis et al. [4], where a bootstrap proportion of $>=70 \%$ usually corresponds to a probability of $>=95 \%$ that the corresponding clade is real. Simplot programme was used to analyse recombinants by bootscanning [5].

\section{Results}

Twenty eight (30\%) patients, including 22 Spanish, were infected with non-B subtypes, with a high diversity: four pure subtypes ( $A, C, G, F 1$ ), four circulating recombinant forms (CRFs) (CRF01_AE, CRF02_AG, CRF12_BF, CRF24_BG) and three unique recombinant forms (URFs) (BG, BF, DB). Subtype $F$ and $B F$ recombinants represented $25 \%$ of non-B subtype viruses. Fifteen (54\%) non-B viruses corresponded to acute and recently acquired infections (subtypes F1, G, CRF24_BG and URF BG).

Of the 38 MSM included in the study, nine (24\%) were infected with non-B viruses (two $\mathrm{F} 1$, two CRF12 BF, one C, one CRF24_BG, one CRF01_AE, one DB and one G). Of the nine MSM with non-B subtypes, seven were Spanish, one was from Argentina (CRF12_BF) and one from Cuba $(G)$.

The phylogenetic tree including transmission clusters is shown in Figure 1. Only bootstrap values $>=70 \%$, considered significant, are indicated in the tree. On the whole, 45 (48\%) patients, including 23 of the 38 MSM (60\%), could be grouped in different clusters. Their characteristics are presented in the Table. It is worth mentioning that the clusters include four patients with acute and five patients with recently acquired infection. 
We identified a total of 17 clusters: one formed by nine patients, four including three individuals and 12 two-person clusters. Although in our study a bootstrap proportion of $>=70 \%$ was considered significant [4], it is to be underlined that all but two clusters were grouped with a bootstrap value close to $100 \%$. The samples X2558 and X2578, corresponding to IDU patients and included in the only cluster with a bootstrap value of $70 \%$, were analysed more in-depth by bootscanning (Figure $2 \mathrm{~A}$ and $\mathrm{B}$ ). One of these samples (X2578) was defined as subtype B strain and the other (X-2558) resulted a BG recombinant with a recombination point at position 2700 (HXB2). In the second plot (Figure 2B) it is shown that subtype $B$ sequence of this $B G$ recombinant grouped with the $B$ sequence of $X-2578$. The fact that both samples only

\section{F I G U R E 1}

Phylogenetic analysis of newly diagnosed HIV cases, Galicia, Spain, 2008-2009

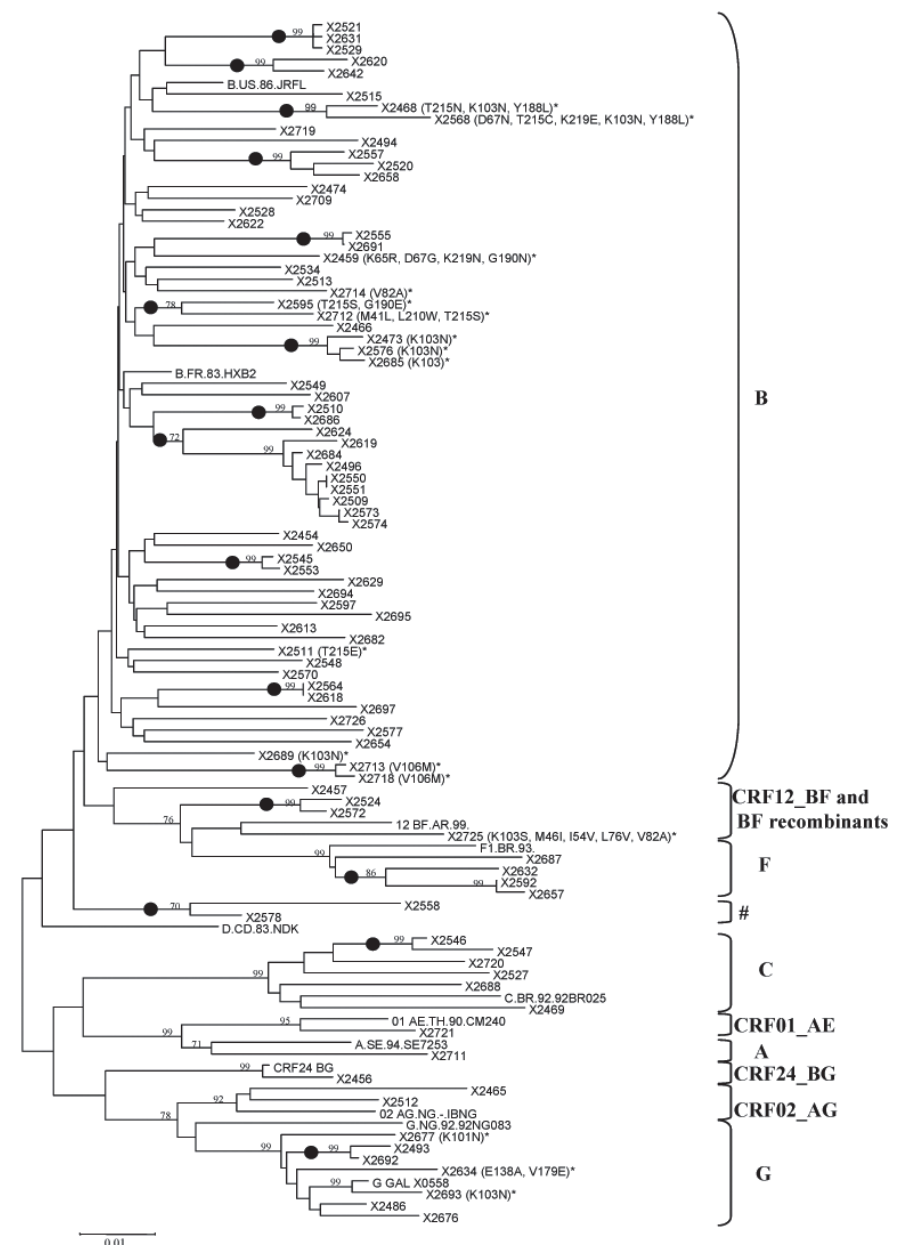

Neighbour-joining algorithm implemented in Molecular Evolutionary Genetic Analysis (MEGA) version 3.1 was used to build the phylogenetic tree of newly diagnosed patients from Galicia. One hundred bootstrap replicates were used to assess the reliability of tree topologies. replicates were used to assess the reliability of tree topologies. Bootstrap values of $70 \%$ or higher, considered as significant, were
included in the tree. Clusters are indicated with dots. Reference of the included in the tree. Clusters are indicated with dots. Reference of the specific subtype $G$ strain which is circulating in Galicia is included with
the name G GAL X0558. Resistance mutations are also included in brackets and highlighted with an asterisk. The \# symbol was used to mark a cluster formed by a BG sample and a B sample as is shown in Figure 2 . The sequence X2556 has been excluded because it showed mixtures. shared a fragment of PR/RT sequence could be the cause of the bootstrap value of $70 \%$.

Of the 23 MSM who were grouped in transmission clusters, it is worth mentioning that seven were included in the large cluster, six in two clusters of three patients and six in two-person clusters, all of them infected with subtype B. The remaining four MSM were grouped with non-MSM patients, as follows: one in a cluster of three individuals infected with subtype $F$, and three in two-person clusters infected with subtype B, G and CRF12_BF, respectively. Most of the MSM who were grouped in transmission clusters attended the same hospital (Xeral-Cies, Vigo, Pontevedra)

Resistance mutations were detected in 17 (18\%) samples: two against nucleoside RT inhibitors (RTIs), nine against non nucleoside RTI (NNRTIs), four against both classes of RTIs, one against PR inhibitors (PRIs), and one against both PRI and NNRTIs. Of the 17 resistance samples, six corresponded to MSM, four subtype $B$, one DB recombinant and one CRF12_BF. Resistance mutations against NNRTIs were detected in three patients, two against NRTIs and NNRTIs and one against NNRTIs and PRIs.

The majority of resistance strains (nine out of 17) were part of the transmission clusters. The most frequent mutation was $\mathrm{K} 103 \mathrm{~N}$, detected in six patients, three of them were MSM included in one transmission cluster.

\section{Discussion}

Of the newly diagnosed HIV-1 patients included in our study, $41 \%$ were MSM, which adds evidence to the observation of increasing transmission among this risk group. This is in line with earlier findings indicating an increase in the percentage of MSM among newly diagnosed HIV cases in Galicia from 31\% in 2006 to $33 \%$ in 2007 (data not published). In another study with 261 newly diagnosed patients from the Basque Country, Spain, we noted an increase in the proportion of MSM from 15.7\% in 2004 to $51.2 \%$ in 2007 [2]. These results are in agreement with studies from other industrialised countries $[3,6]$, underlining that efforts are required to target HIV prevention measures specifically at MSM.

A high prevalence (30\%) of HIV-1 non-B subtypes and a high HIV-1 diversity have been detected in our study, indicating an

T A B L E

Characteristics of HIV patients grouped in transmission clusters based on phylogenetic analysis, Galicia, Spain, 2008-2009

\begin{tabular}{|l|c|c|c|c|c|c|c|}
\hline $\begin{array}{l}\text { HIV } \\
\text { genetic form }\end{array}$ & $\begin{array}{c}\text { Number of } \\
\text { clusters }\end{array}$ & $\begin{array}{c}\text { Number of } \\
\text { patients }\end{array}$ & \multicolumn{3}{|c|}{ Gender } & \multicolumn{4}{|c|}{$\begin{array}{c}\text { Transmission } \\
\text { mode }\end{array}$} \\
\hline & & & M & F & MSM & HT & IDU \\
\hline B & $13^{*}$ & 36 & 29 & 7 & 20 & 9 & 7 \\
\hline G & 1 & 2 & 2 & & 1 & 1 & \\
\hline F1 & 1 & 3 & 1 & 2 & 1 & 2 & \\
\hline C & 1 & 2 & 1 & 1 & & 2 & \\
\hline CRF12_BF & 1 & 2 & 1 & 1 & 1 & 1 & \\
\hline TOTAL & 17 & 45 & 34 & 11 & 23 & 15 & 7 \\
\hline
\end{tabular}

M: male, F: female, MSM: men who have sex with men, HT: heterosexual contact; IDU: injecting drug use.

* One of these clusters is composed by a B strain and a BG recombinant. 


\section{$\underline{\mathrm{X} 2558}$}

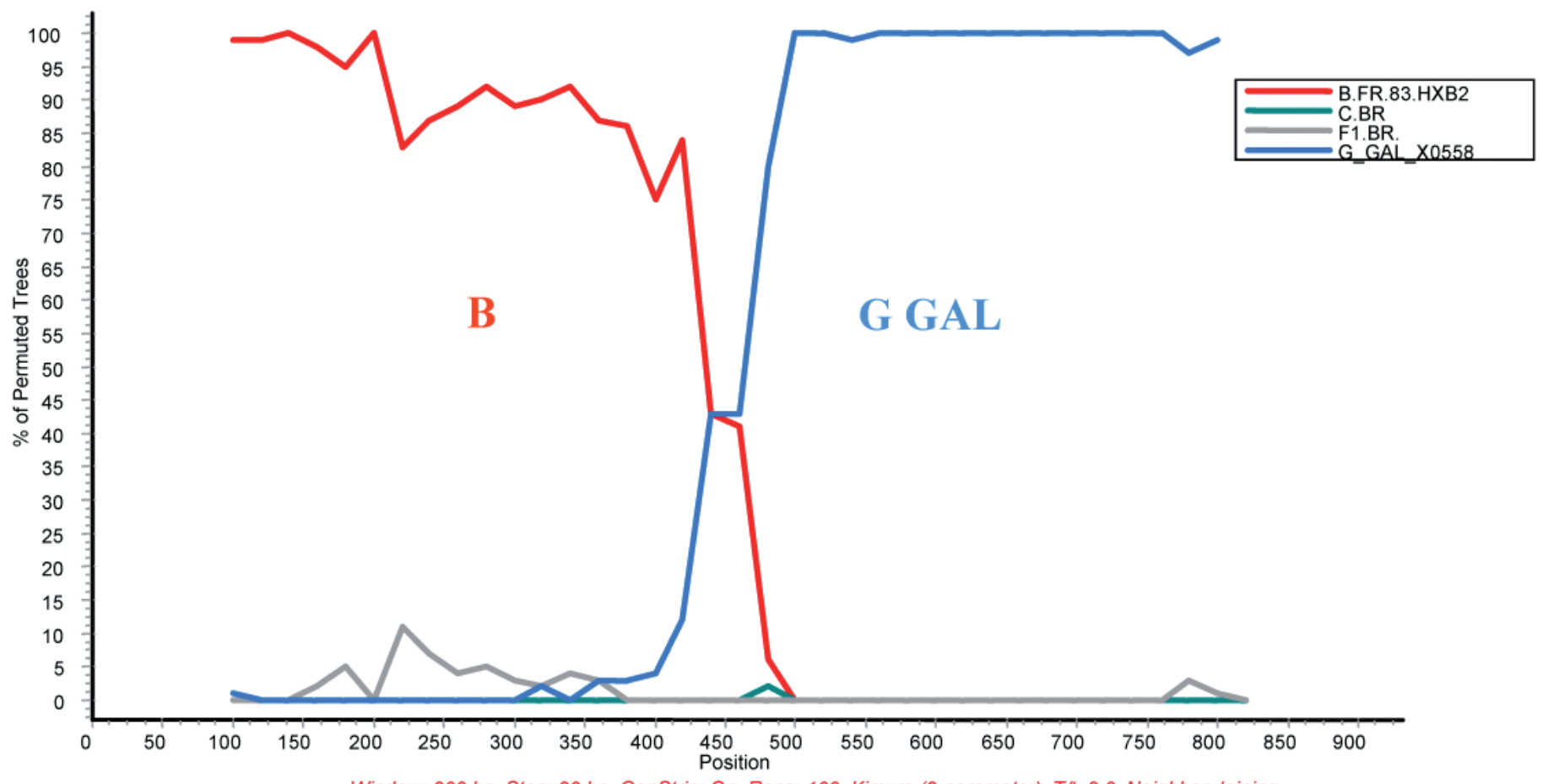

A) The horizontal axis represents the nucleotide position of the midpoint of the window from the $5^{\circ}$ end of protease, and the vertical axis represents the bootstrap value supporting clustering with the corresponding subtype. A window size of 200 nt and a step size of 20 nt was used. Reliability of tree topologies was assessed with 100 bootstrap replicates for each window. Neighbour-joining trees were constructed using Kimura's two-parameter distances. The plot shows a recombination between subtype B and G. The reference subtype G used (G_GAL_X0558) is subtype G circulating in Galicia and distances.

\section{$\underline{\mathbf{X} 2558}$}

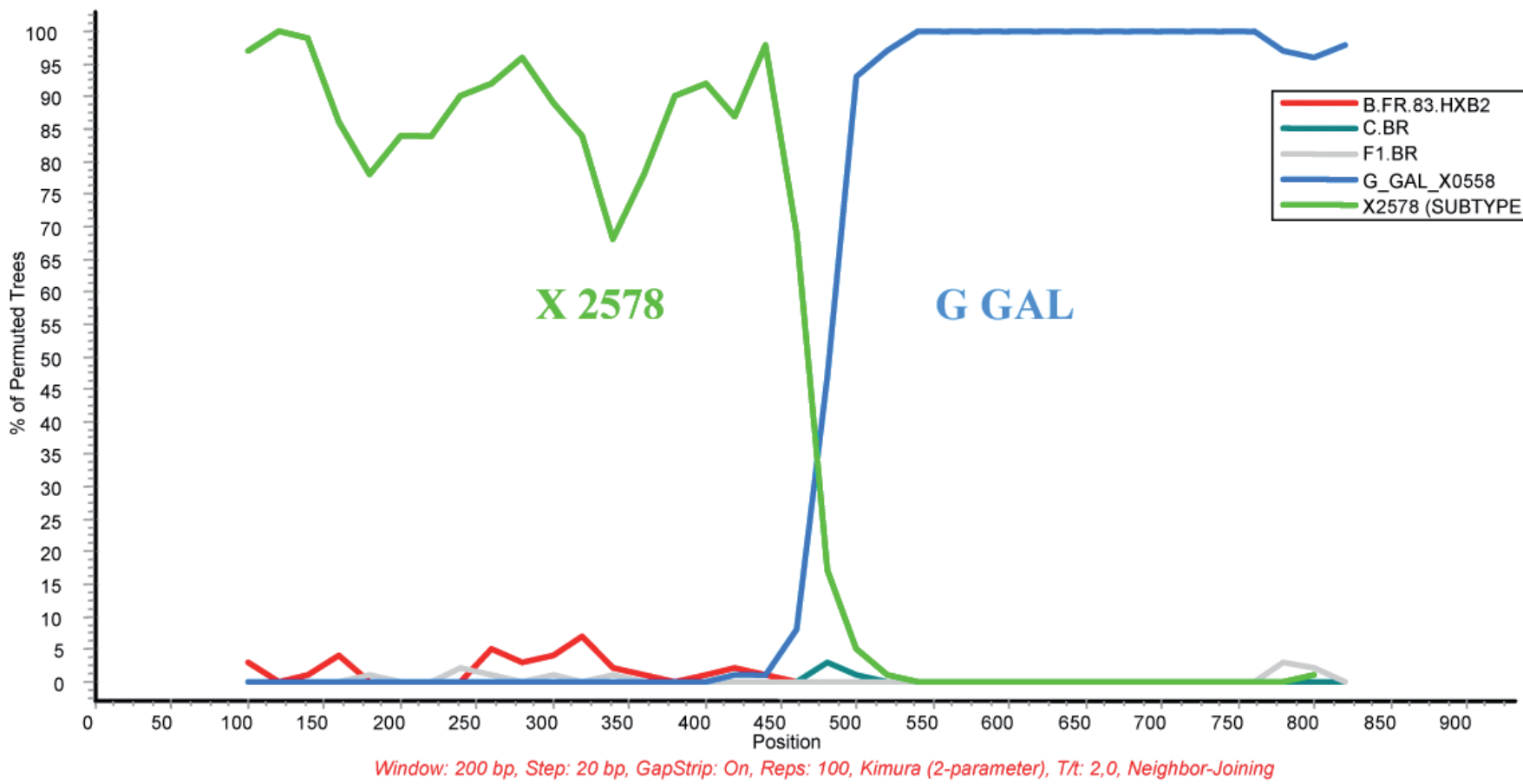

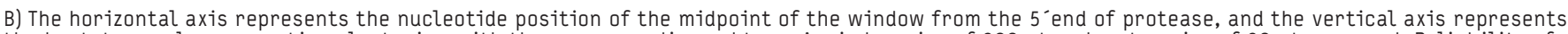
the bootstrap value supporting clustering with the corresponding subtype. A window size of 200 nt and a step size of 20 nt was used. Reliability of tree topologies was assessed with 100 bootstrap replicates for each window. Neighbour-joining trees were constructed using Kimura's two-parameter distances The sequence X2578, corresponding to the sequence clustering with X2558 in the initial analysis, was also used as subtype B reference, showing that the $B$ sequence of $X-2558$ clustered with $\times 2578$ instead of $B$ reference. 
increase in comparison with the results of previous studies in this area $[7,8]$. Most $(79 \%)$ of the non-B subtype strains were found in the native Spanish population. It is worth mentioning that non-B subtypes were also detected in patients with acute or recently acquired infection, indicating that these might be the most commonly viruses circulating in Galicia at the time of this survey.

The proportion of non-B subtypes among MSM (24\%) was similar between Spanish and non-Spanish patients and higher than that described in an Italian survey [9], although in this study a higher frequency among non-Italian patients was detected. In our study, nine different genetics forms were detected, including subtypes, CRFs and URFs. It is worth mentioning the presence of subtype G among MSM, because this subtype was initially transmitted among intravenous drug users [8].

A high proportion of patients, close to half, were phylogenetically clustered. This result is in agreement with the results of Lee et al. [10] whose study focussed on acute and recent drug-naïve seroconverters. It is worth mentioning that 23 out $38(61 \%)$ MSM were grouped in transmission clusters and most frequently in large clusters, whereas clusters detected in heterosexual patients were mostly two-person clusters. Although in our study a bootstrap proportion of $>=70 \%$ was considered significant $[2,4,11]$, it is notable that all but two clusters were grouped with a bootstrap value close to $100 \%$, data that are in agreement with other authors [12], suggesting a common origin of the infection.

One of the most important problems related to newly diagnosed patients is the presence of transmitted resistance mutations which can persist throughout the years, limiting the future treatments [13]. This problem becomes aggravated when newly diagnosed patients and treated patients harbouring resistance mutations $[2,14]$ take part in the same transmission clusters [2,14], facilitating the spread of these resistance strains. In our study most of the resistance strains (nine out of 17) were part of transmission clusters, and three out of six patients with K103N resistance mutation constituted one cluster of three MSM.

In conclusion, our results showed an increase in HIV-1 transmission among MSM, showing a high variability of genetic forms. A high proportion of MSM were grouped in transmission clusters, suggesting an increase in sexual risk behaviour in this group.

Members of the Study group of HIV-1 newly diagnosed patients in Galicia, Spain: Coruña: Hospital Juan Canalejo - MA Castro, MS López-Calvo, JD Pedreira; Hospital del Ferrol - JA Agulla, H Álvarez, A Mariño, P Ordoñez.

Lugo: Hospital Xeral Calde - D Canle, P Alonso, J Corredoira, MJ López-Álvarez.

Ourense: Complejo Hospitalario de Ourense - J García-Costa, R Fernández-Rodríguez, R Rodríguez.

Pontevedra: Complejo Hospitalario Provincial de Pontevedra - S Cortizo, R Ojea de Castro, M Trigo; Complejo Hospitalario Universitario de Vigo - C Miralles, A Ocampo, S Pérez-Castro, A Rodríguez da Silva.

Financial support

This work has been supported by grant FIS PI 080496 (MPY 1416/06-2)

Addendum:

The sequences reported in this article have been sent to GenBank, the accession numbers are: GU326099-GU326190. [added on 7 January 2010.]

\section{References}

1. Madeddu G, Rezza G, Mura MS. Trends in European HIV/AIDS epidemic: a prespective from Italy. Expert Rev Anti Infect Ther. 2009;7(1):25-36.

2. Cuevas MT, Muñoz-Nieto M, Thomson MM, Delgado E, Iribarren JA, Cilla G, et al. HIV-1 transmission cluster with T215D revertant mutation among newly diagnosed patients from the Basque Country, Spain. J Acquir Immune Defic Syndr. 2009;51(1):99-103.

3. Sullivan PS, Hamouda O, Delpech V, Geduld JE, Prejean J, Semaille C, et al. Reemergence of the HIV epidemic among men who have sex with men in North America, Western Europe, and Australia, 1996-2005. Ann Epidemiol. 2009;19(6):423-31.

4. Hillis DM, Bull JJ. An empirical test of bootstrapping as a method for assessing confidence in phylogenetic analysis. Systematic Biology 1993;42(2):182-192.

5. Salminen MO, Carr JK, Burke DS, McCutchan FE. Identification of breakpoints in intergenotypic recombinant of HIV-1 by bootscanning. AIDS Res Hum Retroviruses. 1995;11(11):1423-5.

6. Stall R, Duran L, Wisniewski SR, Friedman MS, Marshal MP, McFarland W, et al. Running in place: implications of HIV incidence estimates among urban men who have sex with men in the United States and other industrialized countries. AIDS Behav. 2009;13(4):615-29.

7. Pérez-Alvarez L, Carmona R, Muñoz M, Delgado E, Thomson MM, Contreras $G$, et al. High incidence of non-B and recombinant HIV-1 strains in newly diagnosed patients in Galicia, Spain: study of genotypic resistance. Antivir Ther. 2003;8(4):355-60.

8. Thomson MM, Delgado E, Manjón N, Ocampo A, Villahermosa ML, Mariño A, et al. HIV-1 genetic diversity in Galicia Spain: BG intersubtype recombinant viruses circulating among injecting drug users. AIDS. 2001;15(4):509-16.

9. Giuliani M, Montieri S, Palamara G, Latini A, Alteri C, Perno CF, et al. Non-B HIV type 1 subtypes among men who have sex with men in Rome, Italy. AIDS Res Hum Retroviruses. 2009;25(2):157-64.

10. Lee C, Sun YJ, Barkham T, Leo YS. Primary drug resistance and transmission analysis of HIV-1 in acute and recent drug-naïve seroconverters in Singapore. HIV Med. 2009;10(6):370-7.

11. Cardoso LP, Queiroz BB, Stefani MM. HIV-1 pol phylogentic diversity and antiretroviral resistance mutations in treated naïve patients from Central West Brazil. J Clin Virol46(2):134-9.

12. Hue S, Clewley JP, Cane PA, Pillay D. HIV-1 pol gene variation is sufficient for reconstruction in the era of antiretroviral therapy. AIDS. 2004;18(5):719-28.

13. Hué S, Gifford RJ, Dunn D, Fernhill E, Pillay D, UK Collaborative Group on HIV Drug Resistance. Demonstration of sustained drug-resistance human immunodeficiency virus type 1 lineages circulating among treatment-naïve individuals. J Virol. 2009;83(6):2645-54.

14. Cuevas MT, Thomson MM, Delgado E, Fernandez-García A, Trigo M, Rodríguez $A$, et al. HIV-1 transmission clusters: a major issue for epidemiological surveillance studies and resistance mutations transmission. Reviews in Antiviral Therapy. 2009;7: 22-23. 